\title{
Application of WeChat Platform to Assist Extended Care for Patients Undergoing Laparoscopic Sleeve Gastrectomy Bariatric Surgery
}

\author{
Li Zhang ${ }^{1}$, Shuqing $\mathrm{Yu}^{2}$, Xiuli Wei ${ }^{2}$, Lina $\mathrm{Wu}^{2}$, Lilian $\mathrm{Gao}^{2}$, * \\ ${ }^{1}$ Department of Comprehensive Ward 1, The First Affiliated Hospital of Jinan University, Guangzhou, China \\ ${ }^{2}$ Department of International Bariatric Center, The First Affiliated Hospital of Jinan University, Guangzhou, China \\ Email address: \\ zhanglijasmine@126.com (Li Zhang),1066267048@qq.com (Shuqing Yu),445827039@qq.com (Xiuli Wei), \\ 1479161420@qq.com (Lina Wu),943309720@qq.com (Lilian Gao) \\ *Corresponding author
}

\section{To cite this article:}

Li Zhang, Shuqing Yu, Xiuli Wei, Lina Wu, Lilian Gao. Application of WeChat Platform to Assist Extended Care for Patients Undergoing Laparoscopic Sleeve Gastrectomy Bariatric Surgery. Journal of Family Medicine and Health Care. Vol. 6, No. 3, 2020, pp. 66-69.

doi: $10.11648 /$ j.jfmhc.20200603.12

Received: May 14, 2020; Accepted: May 29, 2020; Published: June 9, 2020

\begin{abstract}
Purpose: To explore the effect of using WeChat platform to assist extended care on the compliance behaviors, health locus of control and quality of life of patients who were undergoing laparoscopic sleeve gastrectomy (LSG) bariatric surgery. Methods: A total of 120 patients from July 2018 to June 2019 in the First Affiliated Hospital of Jinan University were enrolled in the study. 120 patients were randomly divided into control and intervention groups containing 60 patients respectively. In the control group, patients were given routine extended care. In the intervention group, WeChat platform extended care was adopted in addition to routine extended care. The compliance behaviors of patients in the two groups were collected and compared, and health locus of control and quality of life of patients in the two groups before the intervention and 3 months after the intervention were evaluated using the multidimensional health locus of control scale (MHLC) and 36 item short form health survey (SF-36). Results: The compliance rates of the intervention group were higher than those of the control group $(P<0.05)$. Before the intervention, there were no significant differences between the two groups of patients' health locus of control and quality of life $(P>0.05)$, but 3 months after the intervention, compared with the control group, the health locus of control and quality of life of the intervention group were significantly improved $(P<0.05)$. Conclusions: Application of WeChat platform to assist extended care is beneficial to the enhanced compliance behaviors, improved health locus of control and quality of life of patients undergoing LSG bariatric surgery.
\end{abstract}

Keywords: Extended Care, WeChat Platform, Bariatric Surgery, Compliance Behavior, Quality of Life

\section{Introduction}

Obesity is one of the major diseases currently endangering the health of patients, which can cause cardiovascular and cerebrovascular diseases and metabolic diseases, and seriously affect the health and quality of life of patients [1]. The methods to treat obesity include exercise, diet control, drug therapy and surgical treatment. Surgery is the main clinical method to treat obesity, and minimally invasive laparoscopic sleeve gastrectomy (LSG) surgery is one of the most commonly used bariatric surgical procedures, which has a long-term and stable effect on weight loss and can treat or prevent obesity-related metabolic diseases [2]. More and more obese patients are willing to undergo bariatric surgery. However, LSG bariatric surgery is a kind of gastrointestinal tract reconstruction surgery. Most patients undergoing LSG bariatric surgery are severely obese, and these patients often suffer from psychological problems such as anxiety, depression, eating disorders and addictive personality, which can influence the effect of weight loss [3, 4]. Poor patient compliance with the nursing plan after discharge, such as continuing poor diet, not changing rest and exercise habits, not taking medicine and returning for review on time, are the leading causes of patients' large fluctuations in body mass 
index (BMI) after bariatric surgery [5]. Extended care is an extension of in-hospital nursing to out-of-hospital rehabilitation stage, which can ensure patients' access to continuous health care during recovery, reduce the risk of disease deterioration, reduce the cost of health services and promote patients' recovery. With the development of society and the public's increasing demand for health, extended care has been widely carried out in various hospitals in China $[6,7]$ However, how to improve the effectiveness of extended care has raised higher requirements for our nursing research. In China, WeChat platform has become the most popular social network platform with the popularization of internet and smart mobile phones. Without extra cost and large amount of material and human forces, WeChat has language, text, image, video and other functions as well as simple, economical and practical advantages. Therefore, in this study, using WeChat platform to assist extended care was applied to promote postoperative recovery of patients undergoing LSG bariatric surgery and turned out to be effective. The summary report is as follows.

\section{Data and Method}

\subsection{Research Objects}

120 patients admitted to our hospital's international bariatric center for LSG bariatric surgery from July 2018 to June 2019 were enrolled in this study. The patients were numbered sequentially according to the admission sequence, and then randomly divided into the intervention group of 60 patients and the control group of 60 patients using the random number table method. In the control group, there were 17 males and 43 females with an average age of $29.30 \pm 8.88$ years old, an average BMI of $38.20 \pm 5.81 \mathrm{~kg} / \mathrm{m}^{2}$ and education levels: 1 case with primary school, 25 cases with middle school and 34 cases with university or above. In the intervention group, there were 16 males and 44 females with an average age of $30.77 \pm 8.04$ years old, an average BMI of $37.22 \pm 5.86 \mathrm{~kg} / \mathrm{m}^{2}$ and education levels: 1 case with primary school, 27 cases with middle school and 32 cases with university or above. There were no statistically significant differences between the two groups in general data $(P>0.05)$, suggesting comparability.

Inclusion criteria: (1) patients with a BMI of more than 40 $\mathrm{kg} / \mathrm{m}^{2}$ or a BMI of $28-40 \mathrm{~kg} / \mathrm{m}^{2}$ with more than 2 metabolic diseases, aged 18-55 years old; (2) patients who simply received elective LSG bariatric surgery for the first time; (3) patients who could correctly understand the relevant content of the scale and make answers. Exclusion criteria: (1) patients with previous history of mental illness; (2) patients who had comprehension and hearing impairment and could not complete the scale assessment.

\subsection{Research Methods}

(1) Both groups of patients completed the preoperative examination within the expected time and were treated with an expected LSG bariatric surgery. The control group patients received routine extended care, which was that patients were given routine discharge guidance and postoperative health education sheet before discharge and were followed up by telephone within 10 days, 1 month and 3 months after surgery, respectively.

(2) The intervention group patients received WeChat platform extended care, specific as follows: 1) we established WeChat extended care team and WeChat platform; the WeChat extended care team consisted of 1 nursing manager, 1 nursing team leader and 2 responsible nurses; the nursing manager was responsible for technical guidance; the nursing team leader was responsible for quality control; the responsible nurses were in charge of implementing extended care by WeChat platform; we established "extended care express of international bariatric center" Wechat platform; WeChat platform extended care was conducted for 3 months; 2) we printed the two-dimensional code and functions of the platform on the postoperative health education sheet; before the patients were discharged from the hospital, the responsible nurses guided the patients and their families to scan the two-dimensional code with their mobile phones to join into the platform; at the same time, the care purpose and specific content of the platform were informed to patients; 3 ) with the form of video, picture or text, at least 1 piece of relevant knowledge or chain connection of self-management after bariatric surgery was sent to the patients every day; when the patients have any questions after reading the messages sent by the platform, they could put it forward in the WeChat platform at any time; members of the WeChat platform extended care team will reply regularly in the morning and afternoon by the online interaction and guidance with the patients.

\subsection{Evaluation Index}

In research, the compliance behaviors of patients in the two groups were assessed using a self-designed questionnaire. Health locus of control and quality of life of patients in the two groups before the intervention and 3 months after the intervention were evaluated using the multidimensional health locus of control scale (MHLC) [8] and 36 item short form health survey (SF-36) [9], respectively. MHLC includes 18 items, divided into 3 subscales: the internal health locus of control scale (IHLC) measures how much people believe they can manage their health; the powerful others health locus of control scale (PHLC) measures how much people trust others to control their health; the chance health locus of control scale (CHLC) measures how much people believe that chance factors can affect their health. Each subscale consists of 6 items, rated on a scale of 1 (strongly disagree) to 6 (strongly agree). The total score of each subscale ranged from 6 to 36 points, the higher the score of each subscale, the greater the tendency of the health locus of control. SF-36 includes 8 dimensions of physiological function, role-physical, body pain, general health, vigor, social function, role emotional and mental health. Each dimension contains 2-10 items, a total of 36 
items. The full score of SF-36 is one hundred, the lower the score, the worse the quality of life of the patients.

\subsection{Statistical Methods}

SPSS19.0 software was used for statistical analysis. The measurement data were expressed as $(\bar{x} \pm s)$ and analyzed by $t$ test, and the count data were analyzed by $\chi^{2}$ test. A difference was considered statistically significant at $P<0.05$.

\section{Result}

\subsection{Comparison of the Compliance Behaviors Between the Control Group and Intervention Group}

By comparing the rates of the compliance behaviors of the two groups, it was found the intervention group was statistically significant $(P<0.05)$, as shown in table 1 .

Table 1. Comparison of the compliance behaviors between the two groups [n (\%)].

\begin{tabular}{|c|c|c|c|c|c|}
\hline Group & Cases & Keeping exercising & Being in a good mood & Taking medicine as directed & Eating a reasonable diet \\
\hline Control & 60 & $43(71.67)$ & $44(73.33)$ & $46(76.67)$ & $42(70.00)$ \\
\hline Intervention & 60 & $56(93.33)$ & $57(95.00)$ & $57(95.00)$ & $55(91.67)$ \\
\hline$\chi^{2}$ value & & 9.755 & 10.568 & 8.292 & 9.090 \\
\hline$P$ value & & 0.002 & 0.001 & 0.004 & 0.003 \\
\hline
\end{tabular}

\subsection{Comparison of Scores of MHLC Before and After Intervention Between the Control Group and Intervention Group}

Before the intervention, there were no significant differences between the two groups of patients' scores of MHLC $(P>0.05)$. However, 3 months after the intervention, the PHLC and CHLC scores in the two groups all decreased and the PHLC and CHLC scores in the intervention group were significantly lower than those of the control group $(P<0.05)$; the IHLC scores in both groups increased and the IHLC score in the intervention group was significantly higher than that of the control group $(P<0.05)$, as shown in table 2 .

Table 2. Comparison of scores of MHLC before and after intervention between the two Groups $(\bar{x} \pm s)$.

\begin{tabular}{|c|c|c|c|c|c|c|c|}
\hline \multirow[b]{2}{*}{ Group } & \multirow[b]{2}{*}{ Cases } & \multicolumn{2}{|l|}{ PHLC } & \multicolumn{2}{|l|}{ CHLC } & \multicolumn{2}{|l|}{ IHLC } \\
\hline & & $\begin{array}{l}\text { Before } \\
\text { intervention }\end{array}$ & $\begin{array}{l}\text { After } \\
\text { intervention }\end{array}$ & $\begin{array}{l}\text { Before } \\
\text { intervention }\end{array}$ & $\begin{array}{l}\text { After } \\
\text { intervention }\end{array}$ & $\begin{array}{l}\text { Before } \\
\text { intervention }\end{array}$ & $\begin{array}{l}\text { After } \\
\text { intervention }\end{array}$ \\
\hline Control & 60 & $26.86 \pm 3.43$ & $24.58 \pm 1.25^{*}$ & $17.93 \pm 2.64$ & $17.01 \pm 1.23^{*}$ & $22.35 \pm 5.43$ & $23.92 \pm 2.43^{*}$ \\
\hline Intervention & 60 & $26.75 \pm 2.82$ & $22.13 \pm 6.78^{*}$ & $18.96 \pm 4.45$ & $15.05 \pm 6.45^{*}$ & $21.15 \pm 4.62$ & $26.15 \pm 5.37^{*}$ \\
\hline t value & & 0.192 & 2.753 & 1.542 & 2.312 & 1.304 & 2.931 \\
\hline$P$ value & & 0.848 & 0.007 & 0.126 & 0.023 & 0.195 & 0.004 \\
\hline
\end{tabular}

* Compared with the same group before intervention, the difference was statistically significant $(P<0.05)$.

\subsection{Comparison of Scores of SF-36 Before and After Intervention Between the Control Group and Intervention Group}

Before the intervention, there was no significant difference between the two groups of SF-36 $(P>0.05) .3$ months after the intervention, the scores of SF-36 in the two groups all increased and the score of SF-36 in the intervention group was significantly higher than that of the control group $(P<0.05)$, as shown in table 3.

Table 3. Comparison of scores of SF-36 before and after intervention between the two Groups $(\bar{x} \pm \mathrm{s})$.

\begin{tabular}{llll}
\hline \multirow{2}{*}{ Group } & \multirow{2}{*}{ Cases } & SF-36 & \\
\cline { 3 - 4 } & & Before intervention & After intervention \\
\hline Control & 60 & $53.76 \pm 8.41$ & $82.35 \pm 4.36^{*}$ \\
Intervention & 60 & $54.38 \pm 7.52$ & $87.63 \pm 5.72^{*}$ \\
$t$ value & & 0.426 & 5.687 \\
$P$ value & & 0.671 & $<0.001$ \\
\hline
\end{tabular}

*Compared with the same group before intervention, the difference was statistically significant $(P<0.05)$.

\section{Discussion}

Patients undergoing bariatric surgery during hospitalization may maintain good compliance behaviors, because they can obtain scientific and standardized nursing care; but after discharge from the hospital, lacking of nursing instruction and poor self-management ability contribute to their poor compliance behaviors, which affect surgical outcomes [10]. Health locus of control refers to a generalized expectation of whether the outcome or consequence of an event is controlled by the individual himself or by an external force. According to the theory of locus of control, people with different types of locus of control have different expectations on the outcome of events and take different behaviors [8]. Therefore, effective nursing intervention given to patients undergoing bariatric surgery is helpful to improve patients' compliance behaviors and health locus of control, which are of great significances to help patients to achieve nursing outcomes [11]. The discharge of patients does not mean the termination of nursing care, but the extension of inpatient care, which is a part of the overall nursing care [12]. In terms of caring patients undergoing LSG bariatric surgery, nurses should be aware of the expected outcomes of weight loss care, including long-term processes and lifestyle changes that the patients must participate in to maintain weight loss and improve the quality of life [13].

The results of WeChat platform extended care in this study showed that the rates of the compliance behaviors of the 
intervention group were higher than those of the control group, confirming WeChat platform extended care could not only help them establish recovery confidence and develop good living habits, but also improve patients' compliance behaviors. From the results of the health locus of control after the intervention, it showed that the intervention group tended to IHLC, and the control group tended to PHLC, indicating that the health locus of control of the intervention group was superior to that of the control group. The results of this study also showed that after the intervention, the quality of life of the intervention group was also higher than that of the control group. Those findings are consistent with other studies [14, 15], thus confirming WeChat platform extended care could not only change the routine extended care, which is lack of real-time and continuity, to the WeChat platform extended care, which promotes individual-demanding self-management contents and interactive activities through the platform, which is simple, quick, less restricted, highly targeted and low in cost. It is believed that WeChat platform extended care will be further implemented in the field of postoperative care of bariatric surgery to realize a seamless nursing care process.

\section{Conclusion}

We have adopted WeChat platform to assist extended care for patients undergoing LSG bariatric surgery and achieved positive results in nursing outcomes. WeChat platform extended care ensured the compliance behaviors and improved the health locus of control and quality of life of the patients in their recovery. The limitation of this study is that with the increasing number of patients joining WeChat platform, the number and quality of information requirements were also increasing. However, the application of WeChat to assist extended care is still in the exploratory stage, so professional training is needed to make nurses more skilled before they can complete the corresponding tasks.

\section{Compliance with Ethical Standards}

\section{Conflict of Interests}

The authors declare that they have no conflict of interests.

\section{Statement of Informed Consent}

Informed consent was obtained from all individual participants in this study.

\section{References}

[1] Park, S. (2017). Pathways linking obesity to health-related quality of life. Quality of Life Research, 26, 2209-2218.
[2] Zhao, K. K., Liu, J., Wang, M. C., Yang, H., \& Wu, A. G. (2020). Safety and efficacy of laparoscopic sleeve gastrectomy versus laparoscopic Roux- en-Y gastric bypass: A systematic review and meta-analysis. Journal of Evaluation in Clinical Practice, 26 (1), 290-298.

[3] Lent, M. R., \& Swencionis, C. (2012). Addictive personality and maladaptive eating behaviors in adults seeking bariatric surgery. Eating Behaviors, 13 (1), 67-70.

[4] Dawes, A. J., Maggard-Gibbons, M., Maher, A. R., Booth, M. J., Miake-Lye, I., \& Beroes, J. M. et al. (2016). Mental health conditions among patients seeking and undergoing bariatric surgery: A meta-analysis. JAMA, 315 (2), 150-163.

[5] Ma, C., \& Sun, X. D. (2016). Methods and significance for the determination of gastric volume before and after laparoscopic gastric sleeve resection. Chinese Journal of Minimally Invasive Surgery, 16 (9), 855-857.

[6] Wu, L. X., Ying, Y. P., Luo, X. Y., \& Wang, P. P. (2018). Application effect of triangle hierarchical management model in continuous care of hypertensive patients. Guangxi Medicine, 40 (1), 104-108.

[7] Feng, H. C. (2017). Effect of continuous nursing service model on improving self-management ability and control of blood glucose, blood lipid and blood pressure in patients with type 2 diabetes. Journal of Clinical Medical, 4 (58), 11394-11395.

[8] Wang, X. D., Xi, L., \& Ma, H. (1999). Mental health assessment scale handbook. Chinese Journal of Mental Health (the revised version), 344-345.

[9] Yin, J., Mu, L. Q., Peng, C. J., Xu, L., Mao, Z. Q., \& Zhou, X. J., et al. (2014). Effect of laparoscopic gastric bypass surgery on type 2 diabetes and its effect on quality of life. Chinese Journal of Diabetes, 22 (8), 725-727.

[10] Mu, L. Q., Wang, G., Peng, C. J., Yin, J., Mao, Z. Q., \& Zhou, X. J. (2016). Application effect observation of WeChat platform in the management of patients after bariatric surgery. Qilu Nursing Journal, 22 (6), 40-41.

[11] Aura, P. (2017). Comprehensive care for bariatric surgery patients. AACN Advanced Critical Care, 28 (3), 263-274.

[12] Lin, R., Yang, N. L., Liang, H., \& Liu, R. P. (2019). Research progress of continuous nursing mode in the prevention of nutritional metabolic disorders after gastric bypass surgery. Chinese Journal of Obesity and Metabolic Disease, 5 (03), 177-180.

[13] Janice, N. A., \& Donna, R. W. (2015). Enhancing nursing care by understanding the bariatric patient's journey. AORN Journal, $102(2), 132-140$.

[14] Li, Y. H., Ma, X. W., \& Liang, F. (2019). Application of WeChat small procedure in weight loss management of patients after metabolic surgery. Chinese Journal of Modern Nursing, $26,3348-3352$.

[15] Middleton, K. M. R., Patidar, S. M., \& Perri, M. G. (2012). The impact of extended care on the long-term maintenance of weight loss: A systematic review and meta-analysis. Obesity Reviews, 13 (6), 509-517. 